\title{
Observations of a rotating macrospicule associated with an X-ray jet
}

\author{
S. Kamio ${ }^{1}$, W. Curdt ${ }^{1}$, L. Teriaca ${ }^{1}$, B. Inhester ${ }^{1}$, and S. K. Solanki ${ }^{1,2}$ \\ 1 Max-Planck-Institut für Sonnensystemforschung (MPS), Max-Planck-Str. 2, 37191 Katlenburg-Lindau, Germany \\ e-mail: skamio@spd.aas.org \\ 2 School of Space Research, Kyung Hee University, Yongin, Gyeonggi 446-701, Korea
}

Received 9 September 2009 / Accepted 8 January 2010

ABSTRACT

\begin{abstract}
Aims. We attempt to understand the driving mechanism of a macrospicule and its relationship with a coronal jet. Methods. We study the dynamics of a macrospicule and an associated coronal jet captured by multi-spacecraft observations. Doppler velocities in both the macrospicule and the coronal jet are determined by EIS and SUMER spectra. Their temporal evolution is studied using X-ray and He II $\lambda 304$ images.

Results. A blueshift of $-120 \pm 15 \mathrm{~km} \mathrm{~s}^{-1}$ is detected on one side of the macrospicule, while a redshift of $50 \pm 6 \mathrm{~km} \mathrm{~s}^{-1}$ is found at the base of the other side. The inclination angle of the macrospicule inferred from a stereoscopic analysis with STEREO suggests that the measured Doppler velocities can be attributed to a rotating motion of the macrospicule rather than a radial flow or an expansion. Conclusions. The macrospicule is driven by the unfolding motion of a twisted magnetic flux rope, while the associated X-ray jet is a radial outflow.
\end{abstract}

Key words. Sun: corona - Sun: transition region - Sun: coronal mass ejections (CMEs) - Sun: UV radiation

\section{Introduction}

Macrospicules are spiky features extending from the limb, which were first observed in He II $\lambda 304$ images (Bohlin et al. 1975). They are typically $5^{\prime \prime}$ to $50^{\prime \prime}$ long and $5^{\prime \prime}$ to $30^{\prime \prime}$ wide and have a lifetime of 5 to $40 \mathrm{~min}$. Macrospicules are formed at temperatures lower than $3 \times 10^{5} \mathrm{~K}$ and are not seen in emission at higher temperatures. Since they occur primarily within coronal holes, it has been suggested that open magnetic field lines might play a role in macrospicule formation. Moore et al. (1977) compared images in He II $\lambda 304$ and H $\alpha$ and concluded that $\mathrm{H} \alpha$ macrospicules are the chromospheric counterpart of EUV macrospicules. They showed that flares in X-ray bright points coincided with $\mathrm{H} \alpha$ macrospicules. However, the long exposure time needed for X-ray observations did not allow the temporal evolution to be traced. Habbal \& Gonzalez (1991) reported microwave observations of macrospicules in polar coronal holes. They found pinching-off in the upper part of some macrospicules and the separation of plasmoids. They suggested that macrospicules could supply mass to the solar wind.

Pike \& Mason (1998) reported blueshifted and redshifted emission on opposite sides of macrospicules and interpreted them as rotating motion. However, the problem of spectroscopic observations is that a sequence of spectra is a mixture of spatial structure and temporal evolution because scanning times are often comparable to the lifetime of macrospicules. Considerable morphological evidence of helical signatures in jets has been found (Shimojo et al. 1996; Patsourakos et al. 2008), although the velocity of the helical motion could not be determined.

Apart from macrospicules, it has been reported that numerous X-ray jets occur in polar coronal holes (Cirtain et al. 2007; Savcheva et al. 2007; Shimojo et al. 2007). Their frequent occurrence and the associated high speed outflows of up to $800 \mathrm{~km} \mathrm{~s}^{-1}$ suggest that they may play a role in solar wind acceleration. Many observations detect coronal jets associated with cool features. Canfield et al. (1996) reported the simultaneous occurrence of $\mathrm{H} \alpha$ surges and X-ray jets. Kim et al. (2007) found a correlation of X-ray jets with cooler material showing strong blue shift on the disk. Raouafi et al. (2008) discovered plume haze rising from locations of polar coronal jets.

Our new observations detected the detailed structure and evolution of a macrospicule associated with a coronal jet. The combination of the EUV Imaging Spectrometer (EIS; Culhane et al. 2007a) on Hinode (Kosugi et al. 2007) and Solar Ultraviolet Measurements of Emitted Radiation instrument (SUMER; Wilhelm et al. 1995) on the Solar and Heliospheric Observatory (SOHO; Domingo et al. 1995) allowed us to measure the line of sight (LOS) motions of both macrospicule and coronal jets. At the same time, the X-Ray Telescope (XRT; Golub et al. 2007) on Hinode and SECCHI (Howard et al. 2008; Wülser et al. 2004) on STEREO (Kaiser et al. 2008) traced the evolution of the coronal jet and the macrospicule. In addition, the $3 \mathrm{D}$ geometry of the macrospicule was estimated from a stereoscopic analysis of SECCHI image pairs.

\section{Observations and data reduction}

The northern polar coronal hole was observed by Hinode and SUMER on 4 November 2007. In the coordinated observations, $70^{\prime \prime}$ wide scans were repeatedly carried out by EIS and SUMER at 60 min cadence. This movie mode was intended to increase the probability of capturing an identical event. Strong emission in Fe XII $\lambda 195.12\left(T_{\mathrm{e}}=1.2 \times 10^{6} \mathrm{~K}\right)$ and He II $\lambda 256.32\left(T_{\mathrm{e}}=\right.$ $\left.5.0 \times 10^{4} \mathrm{~K}\right)$ from EIS and Ne VIII $\lambda 770.41\left(T_{\mathrm{e}}=6.3 \times 10^{5} \mathrm{~K}\right)$ and O IV $\lambda 790.20\left(T_{\mathrm{e}}=1.6 \times 10^{5} \mathrm{~K}\right)$ from SUMER were 


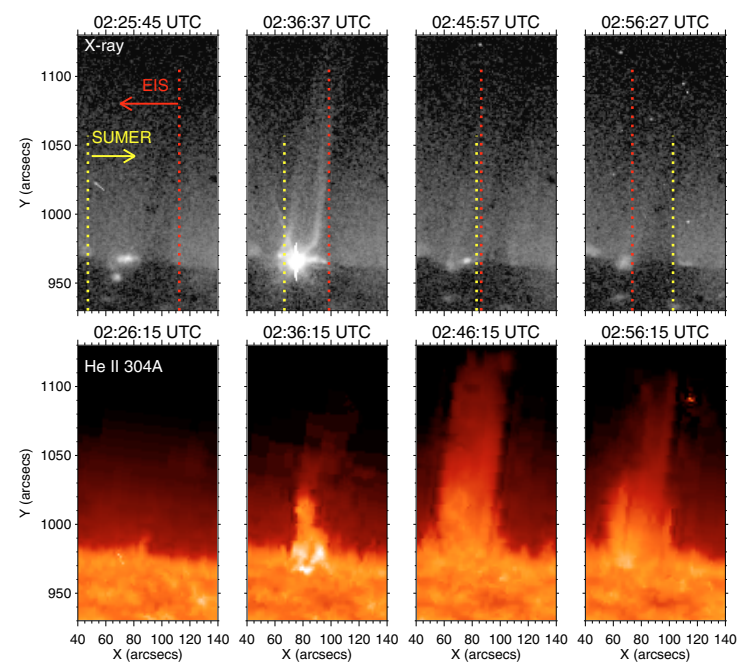

Fig. 1. Top row: sequence of XRT Al_poly filter images showing an $\mathrm{X}$-ray jet. Red and yellow lines respectively indicate EIS and SUMER slit locations at corresponding times. Arrows show scanning directions of EIS and SUMER. Horizontal and vertical axes indicate heliocentric coordinates. Bottom row: STEREO-A EUVI He II $\lambda 304$ images.

selected and analyzed. The exposure time of $45 \mathrm{~s}$ was sufficient to collect enough counts and match the telemetry constraints. EIS and SUMER data were calibrated using standard procedures provided in the Solar Software tree. The spectra were fitted by a Gaussian to derive radiance and Doppler shifts of emission lines.

The $1 \sigma$ value of the Gaussian fit is assumed to measure the uncertainty in the Doppler shift measurements. Velocities are inferred by the assuming that the mean velocity in the quiet region is zero. Co-alignment of EIS and SUMER was checked by identifying bright points in the coronal hole that were stable during the observation. The estimated uncertainty in the alignment is $\approx 1^{\prime \prime}$, the pixel size of EIS and SUMER.

XRT took X-ray images with Al_poly and Ti_poly filters, which are sensitive to low temperature coronal emission down to $1 \times 10^{6} \mathrm{~K}$. The time series with a 1 min cadence allowed us to study the detailed evolution.

SECCHI/EUVI onboard the two STEREO spacecraft recorded He II $\lambda 304$ broadband filter images obtained with a 10 min cadence and $3.2^{\prime \prime}$ resolution. The separation angle of $39^{\circ}$ between STEREO-A and $B$ allowed us to derive the 3D structure of the macrospicule. For the stereoscopic projection of the He II emission from the macrospicule, we separated the macrospicule emission from the image background. The macrospicule images were then divided into 20 equidistant slices of stacked epipolar planes and for each plane the 3D centroid of the observed intensity was constructed. Only the principal axis of the macrospicule was determined since fine structure inside the macrospicule was not resolved in the images. We ignore the slight rotation of the Sun relative to this coordinate system during the $20 \mathrm{~min}$ of the STEREO observations. To obtain accurate results, we had to correct the Sun centre position given in the file headers by a fraction of a pixel (Wülser, private communication). At the time of these observations, these corrections amounted to $\Delta x_{A}=0.0$ pixel, $\Delta y_{A}=-0.7$ pixel, $\Delta x_{B}=0.2$ pixel, and $\Delta y_{B}=0.8$ pixel.

\section{Results}

The sequence of XRT images indicate that five coronal jets occurred at the same location close to the limb between 18:00 UTC on 3 November and 03:00 UTC on the following day. EIS and

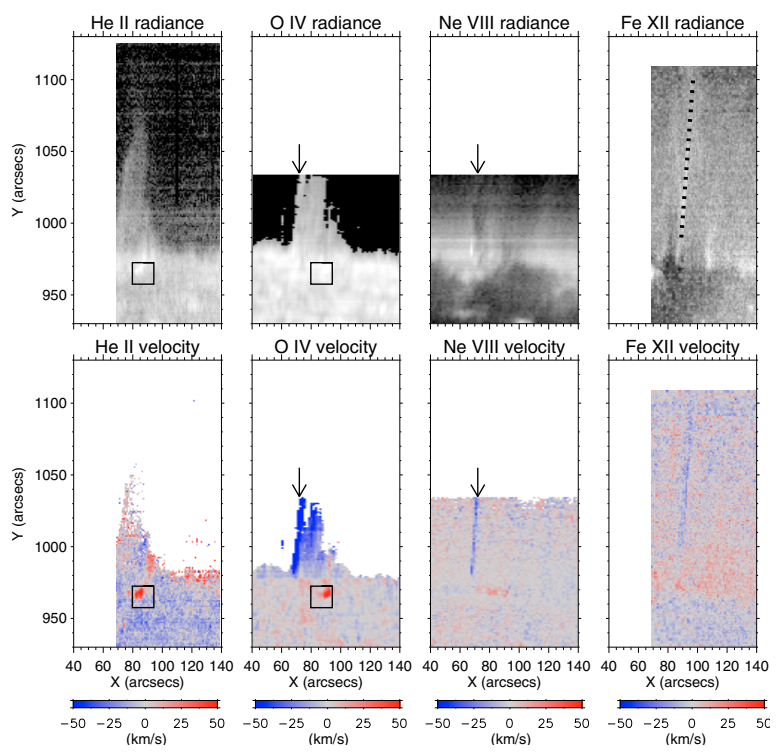

Fig. 2. Radiance and Doppler velocity maps in He II, O IV, Ne VIII, and Fe XII. Boxes in the He II and O IV panels mark the location of the redshifted point. Arrows indicate the location of the blueshifted feature observed by SUMER. Dotted line on the Fe XII image marks the locations of the bright streak on the right side of the macrospicule.

SUMER successfully captured the most significant event hereof. Figure 1 shows time series of X-ray and He II $\lambda 304$ images. Dashed lines plotted on XRT images show the slit locations of EIS and SUMER at the corresponding times. The coronal jet consisted of two threads that originated in an X-ray bright point and extended into the upper corona. The front of the jet reached $8 \times 10^{4} \mathrm{~km}$ above the bright point when it attained maximum brightness at $02: 36$ UTC. The apparent propagation velocity projected on to the plane of the sky is determined by measuring the front height of the jet. The apparent upward velocity inferred from XRT images between 02:31 UTC and 02:36 UTC is $320 \pm 50 \mathrm{~km} \mathrm{~s}^{-1}$.

In He II $\lambda 304$ images at 02:36 UTC, the macrospicule appeared with bright points at the footpoint. The axis of macrospicule deviates by 50 arcsec from the ecliptic pole. If viewed from the direction of the Earth, the estimated displacement is 3 arcsec, which was not compensated for in Fig. 1. EUVI images in Fig. 1 are rotated so that the solar North is up. The axis of the macrospicule is located between the two threads of the X-ray jet. The X-ray jet disappeared by 02:45 UTC, but the macrospicule grew in height and width. The macrospicule attained its maximum height of $1 \times 10^{5} \mathrm{~km}$ at 02:46 UTC, when it expanded to $2.6 \times 10^{4} \mathrm{~km}$ in width. The $10-$ min cadence of He II images is insufficiently short to study the evolution of the macrospicule, but it provides a velocity averaged over $10 \mathrm{~min}$. The upward and horizontal propagating velocities between 02:36 UTC and 02:46 UTC are 130 $\pm 30 \mathrm{~km} \mathrm{~s}^{-1}$ and $25 \pm 5 \mathrm{~km} \mathrm{~s}^{-1}$, respectively. Its upper part became fainter at 02:56 UTC, while the radiance slightly increased at the bottom. The macrospicule had disappeared in the following exposure at 03:06 UTC.

Radiance and Doppler velocities measured by EIS and SUMER are displayed in Fig. 2. The EIS and SUMER slits targeted the jet from opposite directions and successfully captured both edges of the jet at the same time. This is an extremely fortunate case, in which both spectrometers had their slits at the right position and could provide spectroscopic information about a transient, short-lived event. 
The SUMER slit scanned from the left side and enhanced emission in Ne VIII was detected at 02:38 UTC, which is marked in Fig. 2 by arrows in the lower Ne VIII panel. This brightening lies at the foot of a blueshifted streak of about $-25 \pm 5 \mathrm{~km} \mathrm{~s}^{-1}$ observed in Ne VIII, which corresponds to the left thread of the $\mathrm{X}$-ray jet in the second column of Fig. 1.

The excess emission and the blueshift in Ne VIII quickly disappeared as SUMER scanned the inner part of the macrospicule, where the Ne VIII emission was even weaker than the background corona, probably due to absorption by cool dense plasma. Coexisting enhanced emission in O IV indicates the existence of cool material. Anzer \& Heinzel (2005) showed that dark features in EUV coronal emission lines are caused by the presence of cool material in the corona.

At the same time as the blueshifted streak was observed in Ne VIII, offlimb O IV emission appeared, which is unusual for that line. It extended to at least $4.4 \times 10^{4} \mathrm{~km}$ above the limb, which was at the edge of SUMER's FOV. The OIV emission exhibited a blueshift ranging from $0 \pm 10 \mathrm{~km} \mathrm{~s}^{-1}$ at the limb to $-120 \pm 15 \mathrm{~km} \mathrm{~s}^{-1}$ at the top of the slit. As SUMER scanned across to the right, the blueshift gradually decreased and almost vanished at the right edge of the macrospicule.

The EIS slit approached the jet from the right side and encountered enhanced coronal emission in Fe XII at 02:40 UTC, which is marked by a dotted line at $x=90^{\prime \prime}$. The blueshifted streak in Fe XII corresponds to the right thread of the X-ray jet in the second column of Fig. 1. The enhanced emission is blueshifted by $-20 \pm 8 \mathrm{~km} \mathrm{~s}^{-1}$, almost the same velocity as the Ne VIII at $x=70^{\prime \prime}$ on the other side of the structure. On the left side of the enhanced emission, Fe XII radiance became weaker than the surrounding corona, probably because of absorption by cool material, similar to the darkening in Ne VIII.

In the He II velocity, we only studied the ondisk region because the velocity of the offlimb region is largely affected by coronal emission in the red wing of He II $\lambda 256.32$, namely by Si X $\lambda 256.37$, Fe XII $\lambda 256.41$, and Fe XIII $\lambda 256.42$ (Young et al. 2007). Since the He II emission has small a contribution in the offlimb region, increase or decrease in coronal emissions causes an artificial spectral shift. A significant brightening and redshift of $+50 \pm 6 \mathrm{~km} \mathrm{~s}^{-1}$ was found in He II at 02:47 UTC, which is marked by a box in the first column of Fig. 2. Since coronal emission did noticeably change there, the observed redshift can be attributed to He II rather than to blending coronal emission. The SUMER slit reached the region later and also detected a $+50 \pm 4 \mathrm{~km} \mathrm{~s}^{-1}$ redshifted point in O IV at 02:49 UTC, which was located $5 \times 10^{3} \mathrm{~km}$ to the right of the redshift in He II (box in the second column of Fig. 2). The distance of $5 \times 10^{3} \mathrm{~km}$ is greater than the uncertainty in co-alignment, hence it is thought to be a drifting redshifted feature caused by the expansion of the macrospicule seen in Fig. 1. Canfield et al. (1996) studied several $\mathrm{H} \alpha$ surges adjacent to X-ray jets and reported whiplike motion of filaments and moving blueshifted features in $\mathrm{H} \alpha$ surges. The drifting redshifted feature in the present paper could be a similar phenomena.

The reconstructions of the $3 \mathrm{D}$ orientation of the macrospicule were performed in the Heliocentric Earth Equatorial (HEEQ) coordinate system, whose Z-axis is along the Sun's rotation axis and for which zero longitude is the meridian of Earth. For each time, the reconstructed 3D centroid curve was fitted to a principal line. The intersection of this line with the solar surface defined the nominal foot point position of the macrospicule. Table 1 lists the main parameters of the reconstructed macrospicule principal axes at the three STEREO observation times. In Fig. 3, we display these results graphically,
Table 1. Parameters of the macrospicule's principal axis.

\begin{tabular}{cccccc}
\hline \hline Time & \multicolumn{5}{c}{ Footpoint in HEEQ Principal direction Angle to earth } \\
(UTC) & lon $\phi$ & lat $\theta$ & $\Delta \phi$ & $\Delta \theta$ & \\
\hline $02: 36$ & 111.59 & 84.84 & 2.60 & -3.63 & 84.36 \\
$02: 46$ & 113.69 & 85.44 & 4.73 & 2.73 & 84.13 \\
$02: 56$ & 110.05 & 85.63 & 2.49 & 7.89 & 92.37 \\
\hline
\end{tabular}

Notes. The footpoint position is given in HEEQ spherical coordinates The principal direction $\Delta \theta$ and $\Delta \phi$ show the deviations from the radial direction. In the last column, the angle of the principal axis relative to the LOS from the Earth is listed.

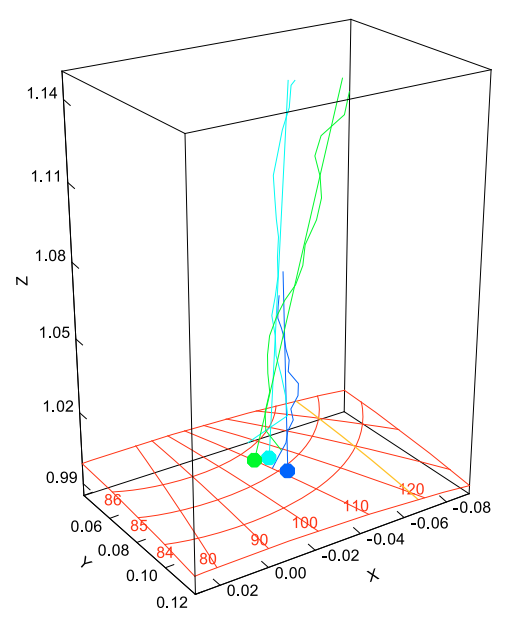

Fig. 3. Oblique view of the macrospicule reconstructed from STEREO SECCHI images. The centroid curve, fitted principal line, and footpoints are colour coded for the three times of observation: 02:36 UTC (dark blue), 02:46 UTC (light blue), 02:56 UTC (green). The Cartesian HEEQ coordinates are given in units of the solar radius along the outer square box. The solar surface is covered with the corresponding spherical grid in red. Earth is in the positive $x$ direction (lower left). The apparent limb from Earth is indicated by the orange curve on the solar surface.

color-coded for the different observation times. The location of the macrospicule is very close to but clearly on the earth-ward side of the apparent limb as seen from Earth. Small values of $\Delta \phi$ and $\Delta \theta$ indicate that the macrospicule axis is close to the radial direction. The last column in Table 1 lists the angle that the reconstructed macrospicule axis makes with the view from Earth, or identically from SUMER and EIS. Since this angle deviates by no more that 6 degrees from 90 degrees, the macrospicule axis is almost perpendicular to the LOS.

\section{Discussion and summary}

Multi-spacecraft observations have monitored the detailed evolution of a macrospicule associated with a coronal jet. The macrospicule exhibited a significant blueshift on the left side and a drifting redshifted feature on the right side, while the coronal jet showed blueshifts on both sides. The 3D reconstruction of the macrospicule has allowed us to distinguish between radial flow and helical motion. Assuming an apparent propagation speed of $130 \pm 30 \mathrm{~km} \mathrm{~s}^{-1}$ corresponding to a radial outflow in the macrospicule, this could account for a LOS velocity of $-15 \mathrm{~km} \mathrm{~s}^{-1}$, which is far smaller than $-120 \pm 15 \mathrm{~km} \mathrm{~s}^{-1}$ observed on the left edge of the macrospicule. Similarly, the apparent horizontal expansion of the macrospicule could only produce a LOS velocity of $-25 \pm 5 \mathrm{~km} \mathrm{~s}^{-1}$. Hence the Doppler shifts 
observed by SUMER can hardly be attributed to a radial outflow or an expansion of the macrospicule. Oppositely directed flows on either side of the macrospicule are indicative of a rotating motion along its axis. The coronal jet exhibited smaller Doppler shifts. If we assume the same inclination angle as for the macrospicule, a radial outflow of $320 \pm 50 \mathrm{~km} \mathrm{~s}^{-1}$, as deduced from the expansion of the jet in a series of X-ray images, could account for a LOS velocity of $-30 \mathrm{~km} \mathrm{~s}^{-1}$, which is comparable to the detected Doppler shifts of $-25 \pm 5 \mathrm{~km} \mathrm{~s}^{-1}$ in Ne VIII and $-20 \pm 8 \mathrm{~km} \mathrm{~s}^{-1}$ in Fe XII. The results suggest that the coronal jet and the macrospicule have different structures; the coronal jet consists of an outflowing hot plasma, while the macrospicule is formed by a cool plasma with rotating motion. The different evolutions of the X-ray jet and the macrospicule also supports this scenario (Fig. 1). The two-threaded X-ray jet travelled at an apparent speed of $320 \pm 50 \mathrm{~km} \mathrm{~s}^{-1}$, while the macrospicule grew at $130 \pm 30 \mathrm{~km} \mathrm{~s}^{-1}$. Nisticò et al. (2009) classified this event as helical by studying a sequence of STEREO/SECCHI images. The He II $\lambda 304$ image at 02:36 UTC (the second column of Fig. 1) shows that the macrospicule has a peculiar shape, which might be interpreted as a twisted flux rope. Fe IX/X $\lambda 171$ images show a pre-existing plume above the jet site. The plume might be connected to the jet, although no noticeable change was found in the plume structure before and after the jet (not shown in the present paper).

Yokoyama \& Shibata (1995) performed two-dimensional MHD simulations and demonstrated that magnetic reconnection between emerging flux and open fields can produce X-ray jets and nearly co-located $\mathrm{H} \alpha$ surges. Both hot and cool plasma are expected to be accelerated more or less at the same time. X-ray jets and neighboring $\mathrm{H} \alpha$ surges were simultaneously observed in active regions (Schmieder et al. 1995). Canfield et al. (1996) observed helical motions of $\mathrm{H} \alpha$ surges and converging magnetic footpoints and interpreted them in the context of Shibata \& Uchida (1986) model in which the successive reconnections release the magnetic twist into open magnetic fields and the twist drives the surge. The same mechanism can also work for the macrospicule. Therefore, it is likely that magnetic reconnection initiated the coronal jet and the macrospicule at the same time.

Pariat et al. (2009) carried out three dimensional MHD simulations in which twist is imposed on axisymmetric magnetic fields. They demonstrated that reconnection between twisted magnetic fields and open fields produces torsional Alfvén waves that drives plasma upflow with helical motion. Here we speculate that magnetic reconnection produced the X-ray jet and the relaxation of helical magnetic fields resulted in the macrospicule. Tian et al. (2008) found that hot and cool loops in a bright point showed different orientations and interpreted them as a twisted loop system. If twisted magnetic loop systems are commonly related to bright points or jets, they could provide the original configuration leading to rotating motion in macrospicules. However, there are still issues to be solved in jet models. The origin of the twist is not fully understood. Pariat et al. (2009) assumed that an axisymmetric field configuration builds up enough energy to produce the helical structure, which may not be realistic.

It seems as if most of the macrospicule falls back to the surface in the end. In the decay phase of the macrospicule, He II indicates enhancement at the bottom and fading out at the upper part (Fig. 1). The fading of the upper part could be caused by heating of cool plasma, but no clear signature of heating was found in hotter emission lines. The radiance enhancement at the bottom can be interpreted as a density increase caused by falling back cool plasma. Culhane et al. (2007b) analyzed slot images obtained with EIS and found post-jet enhancements in cool emission lines, which were also interpreted as falling back jet plasma. Although they did not identify macrospicules as the cause, the delayed enhancement in cool lines including He II that they found could be due to macrospicular material. However, part of the material might be ejected into the corona, because blobs pinching off from macrospicules were reported (Habbal \& Gonzalez 1991).

In our study, both 3D orientation and velocities of a macrospicule have been determined for the first time. These results clearly detect a rotating motion in the macrospicule and show only radial outflow in the coronal jet. Macrospicule and jet models in 3D need to reproduce these properties. Nisticò et al. (2009) carried out a statistical study of coronal jets seen by STEREO/SECCHI and reported many events associated with He II $\lambda 304$ features, including this event. This proves that this event, in which the coronal jet and the macrospicule are initiated at the same time, is a common phenomenon in the solar atmosphere.

Acknowledgements. Hinode is a Japanese mission developed and launched by ISAS/JAXA, with NAOJ as domestic partner and NASA and STFC (UK) as international partners. It is operated by these agencies in co-operation with ESA and NSC (Norway). The SUMER project is financially supported by DLR, CNES, NASA, and the ESA PRODEX Programme (Swiss contribution). SUMER is part of $S O H O$ of ESA and NASA. STEREO is a project of NASA. The data from the SECCHI instrument used here was produced by an international consortion led by the Naval Research Lab (USA). The STEREO contributions by the MPS were supported by DLR grant 50OC0501. This work has been partially supported by WCU grant No. R31-10016 funded by the Korean Ministry of Education, Science, and Technology.

\section{References}

Anzer, U., \& Heinzel, P. 2005, ApJ, 622, 714

Bohlin, J. D., Vogel, S. N., Purcell, J. D., et al. 1975, ApJ, 197, L133 Canfield, R. C., Reardon, K. P., Leka, K. D., et al. 1996, ApJ, 464, 1016 Cirtain, J. W., Golub, L., Lundquist, L., et al. 2007, Science, 318, 1580 Culhane, J. L., Harra, L. K., James, A. M., et al. 2007a, Sol. Phys., 243, 19 Culhane, L., Harra, L. K., Baker, D., et al. 2007b, PASJ, 59, 751 Domingo, V., Fleck, B., \& Poland, A. I. 1995, Sol. Phys., 162, 1 Golub, L., Deluca, E., Austin, G., et al. 2007, Sol. Phys., 243, 63 Habbal, S. R., \& Gonzalez, R. D. 1991, ApJ, 376, L25

Howard, R. A., Moses, J. D., Vourlidas, A., et al. 2008, Space Sci. Rev., 136, 67 Kaiser, M. L., Kucera, T. A., Davila, J. M., et al. 2008, Space Sci. Rev., 136, 5 Kim, Y.-H., Moon, Y.-J., Park, Y.-D., et al. 2007, PASJ, 59, 763 Kosugi, T., Matsuzaki, K., Sakao, T., et al. 2007, Sol. Phys., 243, 3 Moore, R. L., Tang, F., Bohlin, J. D., et al. 1977, ApJ, 218, 286

Nisticò, G., Bothmer, V., Patsourakos, S., et al. 2009, Sol. Phys., 259, 87 Pariat, E., Antiochos, S. K., \& DeVore, C. R. 2009, ApJ, 691, 61 Patsourakos, S., Pariat, E., Vourlidas, A., Antiochos, S. K., \& Wuelser, J. P. 2008, ApJ, 680, L73

Pike, C. D., \& Mason, H. E. 1998, Sol. Phys., 182, 333

Raouafi, N.-E., Petrie, G. J. D., Norton, A. A., Henney, C. J., \& Solanki, S. K. 2008, ApJ, 682, L137

Savcheva, A., Cirtain, J., Deluca, E. E., et al. 2007, PASJ, 59, 771

Schmieder, B., Shibata, K., van Driel-Gesztelyi, L., et al. 1995, Sol. Phys., 156, 245

Shibata, K., \& Uchida, Y. 1986, Sol. Phys., 103, 299

Shimojo, M., Hashimoto, S., Shibata, K., et al. 1996, PASJ, 48, 123

Shimojo, M., Narukage, N., Kano, R., et al. 2007, PASJ, 59, 745

Tian, H., Curdt, W., Marsch, E., et al. 2008, ApJ, 681, L121

Wilhelm, K., Curdt, W., Marsch, E., et al. 1995, Sol. Phys., 162, 189

Wülser, J.-P., Lemen, J. R., Tarbell, T. D., et al. 2004, in SPIE Conf. Ser. 5171, ed. S. Fineschi, \& M. A. Gummin, 111

Yokoyama, T., \& Shibata, K. 1995, Nature, 375, 42

Young, P. R., Del Zanna, G., Mason, H. E., et al. 2007, PASJ, 59, 857 